\title{
ATIVIDADE FÍSICA E ALTERAÇÕES NA HEMOGLOBINA GLI- CADA EM ADOLESCENTES COM DIABETES MELITTUS TIPO 1: QUANTO É NECESSÁRIO? ${ }^{1}$
}

\author{
Valderi Abreu de Lima \\ Universidade Federal do Paraná, Curitiba, Paraná, Brasil \\ Luis Paulo Gomes Mascarenhas \\ Universidade Estadual do Centro-Oeste, Guarapuava, Paraná, Brasil \\ Suzana Nesi França \\ Universidade Federal do Paraná, Curitiba, Paraná, Brasil \\ Juliana Pereira Decimo \\ Universidade Federal do Paraná, Curitiba, Paraná, Brasil \\ William Cordeiro de Souza \\ Universidade do Contestado, Mafra, Santa Catarina, Brasil \\ Neiva Leite \\ Universidade Federal do Paraná, Curitiba, Paraná, Brasil
}

\begin{abstract}
Resumo
O presente estudo teve como objetivo verificar o nível de atividade física de adolescentes com Diabetes Melittus Tipo 1 (DM1) e a correlação com a hemoglobina glicada (HbA1c) e identificar qual quantidade mínima de atividade física moderada a vigorosa (AFMV) é necessária para promover benefícios ao controle glicêmico através da HbA1c. Sendo assim, encontrou-se correlação inversa entre $\mathrm{HbA1c}$ e $\operatorname{AFMV}(\mathrm{r}=-0,34 ; \mathrm{p}=0,04)$ e correlação direta entre $\mathrm{o}$ IMC escore $\mathrm{z}$ e Hba1c $(\mathrm{r}=0,43 ; \mathrm{p}=0,01)$. A curva ROC identificou a área sob a curva (AUC) de $70 \%$ para 45 minutos de AFMV por dia. Dessa forma, adolescentes diabéticos devem realizar no mínimo 45 minutos por dia de AFMV para promover benefícios ao controle glicêmico avaliado por meio da $\mathrm{HbAlc}$.
\end{abstract}

Palavras-chave: Adolescentes. Diabetes Mellitus Tipo1. Atividade motora.

\section{Introdução}

O Diabetes Mellitus Tipo 1 é uma doença autoimune complexa caracterizado por hiperglicemias crônicas resultantes da deficiência da secreção e utilização da insulina (RUBIO-CABEZAS et al., 2014; SBD, 2015). Necessita de um controle glicêmico exógeno com insulinoterapia, cuidados alimentares e a prática de atividades físicas.

\footnotetext{
${ }^{1} \mathrm{O}$ estudo contou com o apoio e Financeiro do CNPq e da CAPES.
} 
As recomendações de Rubio-Cabezas et al., (2014) para um bom controle glicêmico passam pela manutenção dos níveis de hemoglobina glicada (HbA1c) abaixo de 7,5\%. Esse controle glicêmico avaliado pela $\mathrm{HbA1c}$ está associado à diminuição dos fatores de risco para doença cardiovascular e da incidência de complicações como nefropatia, retinopatia, neuropatia diabética e doenças cardiovasculares (GUBITOSI-KLUG, 2014).

A literatura ressalva que médias elevadas de HbA1c estão associadas ao aumento das complicações microvasculares a longo prazo. Desta forma, a manutenção da concentração da hemoglobina glicada inferior a 7,6\% parece ser suficiente para prevenir o desenvolvimento destas complicações por pelo menos 20 anos (NORDWALL et al., 2015).

A atividade física regular desempenha importante papel na prevenção destas complicações a longo prazo em pacientes com DM1(SOUZA et al., 2014). Estudos demonstram uma relação direta entre o controle da glicemia e a prática regular de atividade física e que a diminuição do sedentarismo está associada à melhora do controle da HbA1c (HERBST et al., 2006; RUZIC; SPORIS; MATKOVIC, 2008; AMAN et al., 2009).

Entretanto, é controversa esta relação entre a atividade física e o controle glicêmico. Alguns estudos mostram uma relação inversa entre atividade física e HbA1c (LUKÁCS et al., 2012), enquanto outros trabalhos não demonstraram relação alguma (NADEAU et al., 2010; CUENCA-GARCÍA et al., 2012).

Considerando a hipótese de que a prática diária de atividade física está associada a melhoras nos indicadores de saúde, o presente estudo teve como objetivo verificar se o nível de atividade física de moderada a vigorosa se correlaciona com os níveis de HbA1c e identificar a quantidade de AFMV diária mínima necessária para promover benefícios ao controle glicêmico avaliada por $\mathrm{HbA1c}$.

\section{Materiais e métodos}

Participaram do estudo transversal 44 adolescentes (23 meninos e 21 meninas), com idades entre 10 e 15 anos, atendidos na Unidade de Endocrinologia Pediátrica do Hospital de Clínicas de Curitiba. Todos tinham diagnóstico há mais de seis meses.

Os participantes e seus responsáveis assinaram o termo de assentimento e o Termo de Consentimento Livre e Esclarecido, conforme projeto de pesquisa aprovado pelo Comitê de Ética em Pesquisas com Seres Humanos do Hospital de Clínicas de Curitiba ( $\mathrm{n}^{\circ}$ do parecer: 1.101.601 CAEE: 44193214.7.0000.0096).

A avaliação da estatura foi realizada utilizando estadiômetro vertical portátil (WCS®, Brasil), escalonado em 0,1. A massa corporal foi avaliada com balança digital portátil (Filizola ${ }$, Brasil), com resolução de 100 gramas. O IMC score z foi calculado por meio do programa WHO Antro Plus 10.4. Para a classificação, foi utilizada a referência da escala adotada pelo Sistema de Vigilância Alimentar e Nutricional em 2007 (MINISTÉRIO DA SAÚDE, 2011).

Para a avaliação do nível de atividade física, foi utilizado o questionário de Bouchard et al. (1983), que consiste no registro das atividades diárias do indivíduo a cada 15 minutos, por três dias (dois dias da semana e um do final de semana). Esse instrumento permite estimar o gasto energético pela média dos dias do recordatório.

Os dados do recordatório de atividade física foram anotados de acordo com categorias de 1 a 9 e, assim, foi determinado o tempo gasto em minutos de atividade física dedicado a diversas intensidades. Foram classificados como: tempo de sono (SONO) - categoria 1 do recordatório: atividades sedentárias (AT.SED) - categoria 2: atividades leves (AT.LEVES) categorias 3,4 e 5, e atividades moderadas e intensas (AFMV) - categorias 6,7,8 e 9. 
A hemoglobina glicada (HbA1c) foi verificada por meio do teste imunoturbidimétrico TurbiClin. Após um jejum prévio de 12 horas, os avaliados realizaram pulsão venosa sanguínea. O sangue então foi guardado em tubo de ensaio e levado para análise cromatográfica.

Para a análise dos dados, utilizou-se o programa estatístico SPSS for Windows, versão 22 e MEDcalc®11.6. Para os dados de caracterização da amostra, foi utilizada estatística descritiva com média e desvio-padrão. O teste de correlação de Pearson entre as variável HbA1c, IMC score $\mathrm{Z}$ e o tempo dedicado a atividades nas diversas intensidades foi realizado. Finalmente, a curva ROC foi usada para determinar os pontos de corte em minutos/dia de AFMV necessários para promover benefícios ao controle glicêmico. A área sob a curva ROC determina a acurácia do teste diagnóstico e, quanto maior a área, melhor o resultado (FLETCHER; FLETCHER, 2006). A curva Receiver Operating Characteristic (ROC) é frequentemente utilizada para a determinação de pontos de corte em testes diagnósticos ou de triagem (ERDREICH; LEE, 1981). Para todas as análises, utilizou-se nível de significância p<0,05.

\section{Resultados}

Os dados de caracterização da amostra estão demonstrados na Tabela 1. Do total dos avaliados, 77,27\% $(\mathrm{n}=34)$ tinham HbA1c acima dos valores recomendados de 7,5\% (RUBIOCABEZAS et al., 2014), enquanto apenas $22,72 \%(n=10)$ estavam dentro do recomendado. A insulinoterapia dos avaliados consistiu em doses de insulina de ação prolongada (Glargina) e de ação ultrarrápida (Aspart e Lispro).

Tabela 1. Caracterização da amostra.

\begin{tabular}{lc}
\hline & GDM1 (n=44) \\
\hline Idade (anos) & $12,36 \pm 1,52$ \\
Massa corporal (kg) & $49,12 \pm 11,00$ \\
Estatura (cm) & $154,18 \pm 11,63$ \\
IMC-Z & $0,43 \pm 0,95$ \\
Insulina Glargina (UI) & $26,5 \pm 7,36$ \\
Insulina Aspart e Lispro (UI) & $7,5 \pm 3,41$ \\
HbA1c (\%) & $9,15 \pm 1,61$ \\
\hline \multicolumn{2}{c}{ Fonte: Elaborado pelos autores, 2017.}
\end{tabular}

Os dados do tempo dedicado a diferentes intensidades de atividade física são demonstrados na Tabela 2. O tempo médio dedicado a AFMV foi de 52,74 $\pm 34,11$. Do total de avaliados, apenas 38,63\% $(\mathrm{n}=17)$ alcançaram a recomendação de 60 minutos de AFMV para crianças e adolescentes sugerida pela Organização Mundial da Saúde (2010). Os demais $61,36 \%(n=27)$ não atingiram esse patamar.

Tabela 2. Distribuição do tempo dedicado as diferentes intensidades de atividade física.

\begin{tabular}{lc}
\hline & GDM1 \\
\hline NAF (Kcal/Kg/dia) & $38,85 \pm 4,22$ \\
SONO (horas/dia) & $9,41 \pm 1,42$ \\
AT.SED (minutos/dia) & $631,44 \pm 129,58$ \\
AT.LEVES (minutos/dia) & $174,89 \pm 93,19$ \\
AFMV (minutos/dia) & $52,74 \pm 34,11$ \\
\hline NAF=Nível de atividade física. SONO= Tempo em horas de sono. AT.SED= Tempo dedicado a ativi- \\
dades sedentárias. AT.LEVES= Tempo dedicado a atividades leves. AFMV=Tempo dedicado a ativi- \\
dades de moderada a vigorosa.
\end{tabular}


Fonte: Elaborado pelos autores, 2017.

A análise de correlação entre as variáveis de HbA1c, NAF, horas de sono, atividade sendentária e atividade moderada e vigorosa estão descritas na Tabela 3.

Tabela 3. Correlação das variáveis HbA1c, NAF, horas de sono, atividade sendentaria e atividade moderada evigorosa.

\begin{tabular}{lccccc}
\hline & NAF & SONO & AT.SED & AFMV & IMC-Z \\
\hline HbA1c & $-0,32$ & $-0,01$ & 0,13 & $-0,34^{*}$ & $0,43^{*}$ \\
NAF & 1,00 & $-0,06$ & $-0,54^{* *}$ & $0,90^{* *}$ & $-0,04$ \\
SONO & & 1,00 & $-0,64 * *$ & 0,16 & 0,14 \\
AT.SED & & & 1,00 & $-0,49^{* *}$ & $-0,13$ \\
AFMV & & & & 1,00 & $-0,03$ \\
\hline
\end{tabular}

$\mathrm{HbA1c}=$ Concentração de hemoglobina glicada NAF=Nível de atividade física expresso em Kcal/kg/dia. $\mathrm{SONO}=$ Tempo em horas de sono. AT.SED $=$ Tempo dedicado a atividades sedentárias AFMV=Tempo dedicado a atividades de moderada a vigorosa. Nível de significância $=* \mathrm{p}<0,05 ; * * \mathrm{p}<0,001$. Fonte: Elaborado pelos autores, 2017.

A área sob a curva, valores de sensibilidade e especificidade estão demonstrados na tabela 4 e na Figura 1.

Tabela 4. Ponto de corte com melhor sensibilidade e especificidade do tempo dedicado a AFMV e HbA1c.

\begin{tabular}{cccc}
\hline Área sob a curva & $\begin{array}{c}\text { Ponto de corte } \\
\text { AFMV (min/dia) }\end{array}$ & Sensibilidade & Especificidade \\
\hline 0,701 & 45 & 66,67 & 72,73 \\
\hline AFMV= atividade física de intensidade moderada a vigorosa. & \\
\hline
\end{tabular}

Fonte: Elaborado pelos autores, 2017.

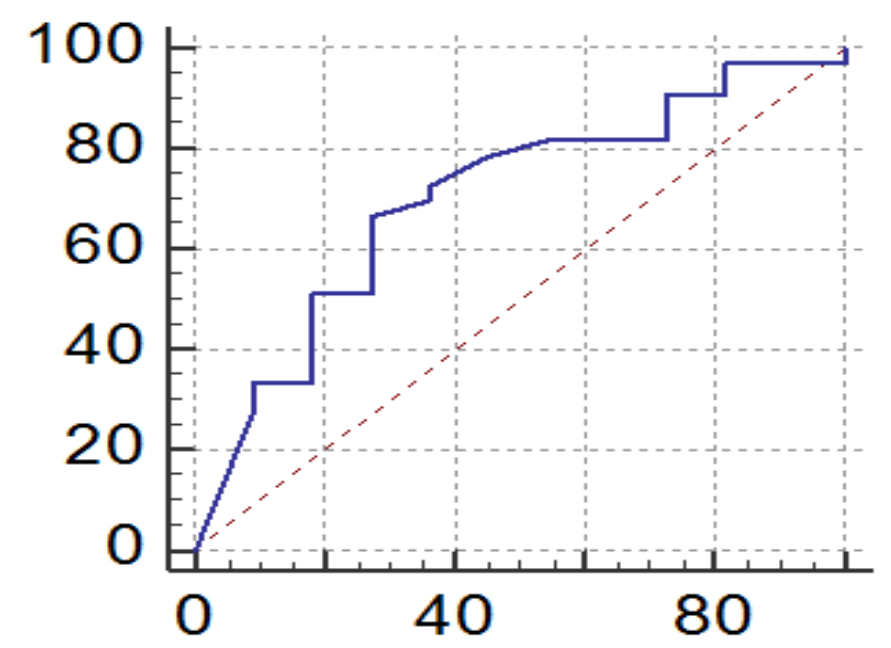

Figura 1. Área sobre a Curva ROC entre AFMV e HbA1c.

Fonte: Elaborado pelos autores, 2017.

\section{Discussão}

A maioria dos avaliados no presente estudo (75\%) encontrava-se em estado eutrófico pela classificação do IMC escore z para idade e peso, enquanto $25 \%$ estavam acima do peso (Tabela 1). Em estudo sobre prevalência de sobrepeso e obesidade em adolescentes com DM1 
realizado no Rio de Janeiro, com 170 pacientes, observou-se uma prevalência de 21,2\% de sobrepeso e obesidade, resultado semelhante ao presente estudo (MORAES et al., 2003).

Um bom controle glicêmico em DM1 recomenda a concentração da HbA1c abaixo de 7,5\% (RUBIO-CABEZAS et al., 2014). Entretanto, 77,27\% dos avaliados apresentaram valores acima da recomendação. Os valores médios de HbA1c observados foram de 9,15\% 1,61, o que demonstra um controle glicêmico inadequado (Tabela 1). Nesta fase da vida, ocorrem muitas mudanças biológicas e psíquicas nos adolescentes. De maneira geral, eles têm maior resistência insulínica que os adultos, com aumento das respostas dos hormônios contrarreguladores, dificultando, assim, a ação da insulina e o controle metabólico (AMIEL et al., 1986; AMIEL et al., 1987).

O tempo médio dedicado a atividades de moderada a vigorosa (AFMV) foi de 52,74 minutos diários, abaixo da quantidade recomendada para crianças e adolescentes (ORGANIZAÇÃO MUNDIAL DA SAÚDE, 2010). Em proporção, 38,63\% (n=17) alcançaram a quantidade recomendada, valores estes superiores aos relatados por Schweiger, Klingensmith e Snell-Bergeon (2010), que observaram que apenas 5\% das meninas com DM1 alcançaram a recomendação internacional. Porém, o resultado é abaixo dos valores encontrados em estudo que avaliou adolescentes sem a doença, no município de Londrina - PR, no qual os dados coletados mostraram que $54 \%$ dos meninos avaliados foram considerados ativos (GUEDES et al., 2001).

Os resultados da Tabela 3 demonstram correlação inversa entre HbA1c e AFMV ( $r=-$ $0,34 ; \mathrm{p}=0,04)$, indicando que, quanto mais as crianças e os adolescentes se envolverem em atividades físicas de intensidade moderada a vigorosa, melhor é o controle glicêmico. A atividade física parece ter a capacidade de aumentar a captação de glicose pelas células musculares, além de aumentar a sensibilidade à insulina, que também é presente no DM1 (KOIVISTO; YKI-JÄRVINEN; DEFRONZO, 1986), favorecendo a esta relação inversa entre o controle glicêmico e a HbA1c. Nas demais variáveis, não foi encontrada significância entre elas.

Valério et al. (2007) analisaram 138 crianças e adolescentes, comparando o controle glicêmico em dois grupos, ativos e não ativos. Os resultados encontrados demonstraram um pior controle glicêmico em pacientes inativos quando comparados a seus pares fisicamente ativos.

Porém, a relação entre a AF e o controle glicêmico ainda é controversa, pois outros estudos não encontraram associação entre as variáveis (NADEAU et al., 2010; CUENCAGARCIA et al., 2012).

Foi observada no presente estudo a relação entre o IMC escore z e Hba1C ( $r=0,43$; $\mathrm{p}=0,01$ ). De acordo com os resultados, o aumento dos índices de IMC escore $\mathrm{z}$, ou seja, o pior controle nutricional pode levar a piores resultados na hemoglobina glicada. É importante a manutenção de um bom controle glicêmico e a concentração de HbA1c igual ou abaixo de $7,5 \%$, uma vez que valores constantes de hemoglobina glicada acima de 10,4\% estão associados a um aumento de até sete vezes do risco de mortalidade por complicações cardiovasculares em DM1.

O ponto de corte para a quantidade de atividade física determinada pela análise da curva ROC reporta que os adolescentes diabéticos precisam realizar pelo menos 45 minutos de AFMV por dia para manter um estilo de vida que promova benefício ao controle glicêmico adequado avaliado por HbA1c. A área sob a curva (AUC) avaliada foi de 70\%, com intervalo de confiança de 54 a 83 e nível de confiança de $95 \%$.

A análise realizada pelo presente estudo sugere que, com um tempo de atividade física de moderada a vigorosa diária de no mínimo 45 minutos, já é possível obter um melhor controle glicêmico em pacientes com diabetes tipo 1, demonstrando a importância de pacientes diabéticos terem um estilo de vida ativo desde a infância e a adolescência. 
Até o momento, os autores não identificaram nenhum estudo que dimencionasse a quantidade de atividade física em diabéticos tipo 1 necessária para exercer influência sobre a hemoglobina glicada de adolescentes. A influência da atividade física sobre a concentração dahemoglobina glicada em portadores de DM1 parece levar a um melhor controle glicêmico. Alguns estudos demonstram a diminuição dos níveis de HbA1c após um programa regular de atividade física (BERNARDINI et al., 2004; MOSHER et al., 1998).

A manutenção de um estilo de vida ativo, com a prática regular de exercícios físicos, está associada a um melhor controle glicêmico e metabólico (ZOPPINI; CARLINI; MUGGEO, 2003). Benefícios no perfil lipídico e redução dos fatores de risco cardiovasculares também são relatados em estudos sobre a prática de exercícios, apontando melhoras na aptidão cardiorrespiratória (AUSTIN et al., 1993).

O estudo apresenta algumas limitações. Os dados devem ser usados com cautela, em virtude do pequeno número amostral e pelo fato de a característica transversal do estudo não permitir uma relação de causa e efeito. O instrumento de avaliação do nível de atividade física utilizado por questionário pode ter um viés de memória, porém, isso não invalida os resultados, pois o método de avaliação é bastante fidedigno para a avaliação do nível de atividade física para crianças e adolescentes com uma correlação de $r=0,74$ e $r=0,79$ e reprodutibilidade a partir de 10 anos de idade (r=0,91) (GUEDES et al., 2001; MASCARENHAS et al., 2005).

Futuros estudos identificando se 45 minutos de atividade física diárias são realmente efetivos para melhora na HbA1c são necessários, para, assim, dimensionar a influência da atividade física e um melhor controle glicêmico de pacientes com diabetes tipo 1.

\title{
Conclusão
}

Os resultados deste estudo reforçam evidências de que a manutenção do peso corporal nos níveis adequados avaliado por IMC escore $\mathrm{z}$ e a prática de atividades físicas, principalmente de intensidade moderada a vigorosa, estão associadas ao melhor controle glicêmico. Com base nas análises realizadas, sugere-se que crianças e adolescentes devem realizar no mínimo 45 minutos por dia de AFMV para promover benefícios ao controle glicêmico avaliado por meio da HbA1c.

\section{PHYSICAL ACTIVITY AND CHANGES IN GLYCATED HEMOGLOBIN IN TEEN- AGERS WITH DIABETES MELLITUS TYPE 1: HOW MUCH IS NEEDED?}

\begin{abstract}
This study aimed to verify the level of physical activity of adolescents with diabetes mellitus type 1 (DM1) and the correlation with glycated hemoglobin (HbA1c), and identify the minimum amount of moderate to vigorous physical activity (MVPA) is required to promote benefits in glycemic control by HbA1c. Thus, found an inverse correlation between HbA1c and MVPA ( $\mathrm{r}=-0.34 ; \mathrm{p}=0.04)$ and direct correlation between BMI z score Hba1c $(\mathrm{r}=0.43 ; \mathrm{p}=$ 0.01 ), the ROC curve identified the area under the curve (AUC) from $70 \%$ to 45 minutes MIPA per day. Thus, diabetic patients should perform at least 45 minutes per day of MVPA to promote benefits in glycemic control assessed by HbA1c.
\end{abstract}

Keywords: Teens. Type 1 Diabetes Mellitus. Motor activity. 


\section{ACTIVIDAD Y LOS CAMBIOS EN LA HEMOGLOBINA GLUCOSILADA EN LOS ADOLESCENTES CON DIABETES TIPO 1 MELLITUS FÍSICO: ¿CUÁNTO SE NECESITA?}

\section{Resumen}

Este estudio tuvo como objetivo verificar el nivel de actividad física de los adolescentes con diabetes mellitus tipo 1 (DM1) y la correlación con la hemoglobina glucosilada (HbA1c), e identificar la cantidad mínima de actividad física moderada a vigorosa (MVPA) para promover beneficios en el control glucémico mediante la HbA1c. Por lo tanto, se encuentra una correlación inversa entre la HbA1c y moderada a vigorosa $(\mathrm{r}=-0,34 ; \mathrm{p}=0,04)$ y la correlación directa entre el IMC z puntuación de $\mathrm{HbA1c}(\mathrm{r}=0,43 ; \mathrm{p}=0,01)$, la curva ROC identificó el área bajo la curva (AUC) de 70\% a 45 minutos MIPA por día. Por lo tanto, los pacientes diabéticos deben realizar al menos 45 minutos diarios de moderada a vigorosa para promover beneficios en el control glucémico evaluado por la HbA1c.

Palabras-clave: Adolescentes. Diabetes Mellitus Pipo 1. Actividad motora.

\section{Referências}

AMAN, J.; SKINNER, T. C.; BEAUFORT, C. E.; SWIFT, P. G.; AANSTOOT, H. J.; CAMERON, F. Associations between physical activity, sedentary behavior, and glycemic control in a large cohort of adolescents with type 1 diabetes: the Hvidoere Study Group on Childhood Diabetes. Pediatric Diabetes, v. 10, n. 4, p. 234-239, 2009.

AMIEL, S. A.; SHERWIN, R. S.; SIMONSON, D. C.; LAURITANO, A. A.; TAMBORLANE, W. Impaired insulin action in puberty: a contributing factor to poor glycemic control in adolescents with diabetes. The New England Journal of Medicine, v. 315, n. 24, p. 215219, 1986.

AMIEL, S. A.; SIMONSON, D. C.; SHERWIN, R. S.; LAURITANO, A. A.; TAMBORLANE, W. Exaggerated epinephrine responses to hypoglycemia in normal and insulindependent diabetic children. The Journal of Pediatrics, v. 110, n. 6, p. 832-837, 1987.

AUSTIN, A.; WARTY, V.; JANOSKY, J.; ARSLANIAN, S. The relationship of physical fitness to lipid and lipoprotein(a) levels in adolescents with IDDM. Diabetes Care, v. 16, n. 2, p. 421-425, 1993.

BERNARDINI, A. L.; VANELLI, M.; CHIARI, G.; IOVANE, B.; GELMETTI. C.; VITALE, R.; ERRICO, M. K. Adherence to physical activity in young people with type 1. Acta Biomedica, v. 75, n. 3, 153-157, 2004.

BOUCHARD, C. A.; TREMBLAY, A.; LEBLANC, C.; LORTIE， G.; SAVARD, R.; THÉRIAULT. G. A method to assess energy expenditure in children and adults. American Journal of Clinical Nutrition, v. 37, n. 3, p. 461-467, 1983.

CUENCA-GARCÍA, M.; JAGO, R.; SHIELD, J. P.; BURREN, C. P. How does physical activity and fitness influence glycemic control in young people with Type 1 diabetes? Diabetic Medicine, v. 29, n. 10, p. 369-376, 2012. 
ERDREICH, L. S.; LEE, E. T. Use of relative operating characteristics analysis in epidemiology: a method for dealing with subjective judgement. American Journal of Epidemiology, v. 114 , n. 5 , p. $649-462,1981$.

FLETCHER, R. H.; FLETCHER, S. W. Diagnóstico curva ROC. In: FLETCHER, R. H.; FLETCHER, S. W. Epidemiologia clínica elementos essenciais. 4. ed. Porto Alegre: Artmed, 2006 p. 63-64.

GUBITOSI-KLUG, R. A. The diabetes control and complications trial/epidemiology of diabetes interventions and complications study at 30 years: summary and future directions. Diabetes Care, v. 37, n. 1, p. 44-49, 2014.

GUEDES, D. P.; GUEDES, J. E. R. P.; BARBOSA, D. S.; OLIVEIRA, J. A. Níveis de pratica de atividade física em adolescentes. Revista Brasileira de Medicina do Esporte, v. 7, n. 6, p.187-199, 2001.

HERBST, A.; BACHRAN, R.; KAPELLEN, T.; HOLL, R. W. Effects of regular physical activity on control of glycemia in pediatric patients with type 1 diabetes mellitus. Archives of Pediatrics and Adolescent Medicine, v. 160, n. 6, p. 573-577, 2006.

KOIVISTO, V. A.; YKI-Järvinen, H.; DEFRONZO, R. A. Physical training and insulin sensitivity. Diabetes/Metabolism Research and Reviews, v. 1, n. 4, p. 445-481, 1986.

LUKÁCS, A.; MAYER, K.; JUHÁSZ, E.; VARGA, B.; FODOR, B.; BARKAI, L. Reduced physical fitness in children and adolescents with type 1 diabetes. Pediatrics Diabetes, v. 13, n. 5, p. 432-337, 2012.

MASCARENHAS, L. P. G.; SALGUEIROSA, F. M.; NUNES, G. F.; MARTINS, P. Â.; STABELINI NETO, A.; CAMPOS, W. Relação entre diferentes índices de atividade física e preditores de adiposidade em adolescentes de ambos os sexos. Revista Brasileira de Medicina do Esporte, v. 11, n. 4, p. 214-218, 2005.

MINISTÉRIO DA SAÚDE. Política nacional de nutrição e alimentação. Brasília, DF: Ministério da Saúde, 2011.

MORAES, C. M. M.; PORTELlA, R. B. P.; PINHEIRO, V. S.; OLIVEIRA, M. M. S.; FUCKS, A. G.; CUNHA, E. F; GOMES, M. B. Prevalência de sobrepeso e obesidade em pacientes com diabetes tipo 1. Arquivos Brasileiros de Endocrinologia e Metabologia, v. 47, n. 6, p. 677-683, 2003.

MOSHER, P. E.; NASH, M. S.; PERRY, A. C.; LAPERRIERE, A. R.; GOLDBERG, R. B. Aerobic circuit exercise training: effect on adolescents with wellcontrolled insulin-dependent diabetes mellitus. Archives of Physical Medicine and Rehabilitation, v. 79, n. 6, p. 652657, 1998.

NADEAU, K. J.; REGENSTEINER, J. G.; BAUER, T. A.; BROWN, M. S.; DOROSZ, J. L.; HULL, A.; ZEITLER, P.; DRAZNIN, B.; REUSCH, J. E. Insulin resistance in adolescents with type 1 diabetes and its relationship to cardiovascular function. The Journal of Clinical Endocrinoloy Metabolism, v. 95, n, 2, p. 513-521, 2010. 
NORDWALL, M.; ABRAHAMSSON, M.; DHIR, M.; FREDRIKSON, M.; LUDVIGSSON, J.; ARNQVIST, H J. Impact of HbA1c, followed from onset of type 1 diabetes, on the development of severe retinopathy and nephropathy: the VISS study (Vascular Diabetic Complications in Southeast Sweden). Diabetes Care, v. 38, n. 2, p. 308-315, 2015.

Organização Mundial da Saúde. Recomendações globais sobre atividade física para a saúde. Genebra: Organização Mundial de Saúde - OMS, 2010, p. 8-10. Disponível em: $<$ http://www.who.int/dietphysicalactivity/publications/9789241599979/en/index.html> Acesso em: 20 maio 2016.

RUBIO-CABEZAS, O.; HATTERSLEY, A. T.; NJØLSTAD, P. R.; MLYNARSKI, W.; ELLARD, S.; WHITE, N.; CHI, D. V.; CRAIG, M. E. The diagnosis and management of monogenic diabetes in children and adolescentes. Pediatric Diabetes, v. 15, n. Supplement 20, p. 47-64, 2014.

RUZIC, L.; SPORIS, G.; MATKOVIC, B. R. High volume-low intensity exercise camp and glycemic control in diabetic children. Journal of Pediatrics and Child Health, v. 44, n. 3, p. 122-128, 2008.

SCHWEIGER, B.; KLINGENSMITH, G.; SNELL-BERGEON, G. K. Physical activity in adolescent females with type 1 diabetes. International Journal of Pediatrics, v. 2010, p. 16, 2010.

SOCIEDADE BRASILEIRA DE DIABETES (SBD). Atividade física e diabetes: a prática segura de atividades desportivas. 2015. Disponível em: <www.diabetes.org.br> Acesso em: 5 maio 2016.

SOUZA, A. C. R. M.; SILVA, D. C.; FREITAS, T. G. P.; BORGES, M. F. Avaliação do nível de atividade física em adolescentes com diabetes mellitus tipo 1 e sua correlação com variáveis metabólicas. Revista Brasileira de Atividade Física e Saúde, v. 19, n. 1, p. 109120, 2014.

VALÉRIO, G.; SPAGNUOLO, M. I.; LOMBARDI, F.; S`PADARO, R.; SIANO, M.; FRANZESE, A. Physical activity and sports participation in children and adolescents with type 1diabetes mellitus. Nutrition, Metabolism\& Cardiovascular Diseases, v. 17, n. 5, p. 376-382, 2007.

ZOPPINI, G.; CARLINI, M.; MUGGEO, M. Self-reported exercise and quality of life in young type 1 diabetic subjects. Diabetes Nutrition and Metabolism, v. 16, n. 1, p. 77-80. 2003.

Recebido em: 22/04/2016

Revisado em: 11/07/2016

Aprovado em: 01/09/2016

Endereço para correspondência:

williammixx@hotmail.com

William Cordeiro de Souza

Universidade do Contestado

Av. Presidente Nereu Ramos, 1071, Jardim do Moinho, 89300-000 - Mafra, SC - Brasil 